\title{
CKD-EPI is a Better Tool for Detecting Renal Dysfunction in Hypertensive Pregnancy: A Case-Control Study in Ghana
}

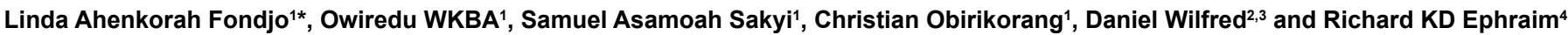

${ }^{1}$ Department of Molecular Medicine, School of Medical Sciences, College of Health Sciences, Kwame Nkrumah University of Science and Technology, Kumasi, Ghana ${ }^{2}$ Department of Medical Laboratory Technology, College of Health Sciences, Faculty of Allied Health Sciences, Kwame Nkrumah University of Science and Technology, Kumasi, Ghana

${ }^{3}$ Medilab Diagnostics Services Ltd, Adum, Kumasi, Ghana

${ }^{4}$ Department of Medical Laboratory Technology, School of Allied Health Sciences, College of Health and Allied Sciences, University of Cape Coast, Cape Coast, Ghana

\begin{abstract}
Background: Pregnant women with hypertension are at an increased risk of renal impairment. This study assessed the use of CKD-EPI and 4V-MDRD for early diagnosis of renal impairment in pregnant women with
\end{abstract} hypertension.

Methods: This case-control study was conducted at Suntreso Government Hospital Kumasi, Ghana. In all, 220 pregnant women were recruited, 84 had Gestational Hypertension, 36 had Preeclampsia, with 100 normotensive pregnant women as controls. Structured Questionnaires were used to obtain socio-demographic and clinical information. $4 \mathrm{~mL}$ of venous blood was collected for estimation of electrolytes, urea, creatinine and uric acid; urine was collected for estimation of protein using dipstick. The Chronic Kidney Disease Epidemiology Collaboration (CKD-EPI) and Modification of Diet in Renal Disease (MDRD-4) equations were used to assess and classify renal impairments.

Results: Prevalence of renal impairment using CKD-EPI and MDRD-4 was $4.1 \%$ and $0.5 \%$ respectively. CKDEPI identified $22.2 \%$ of women with preeclampsia as having renal impairment whereas MDRD-4 identified $2.8 \%$. Using CKD-EPI and MDRD-4, eGFR was significantly higher in the controls compared to the cases, $(p<0.001)$. Sodium, chloride, urea, creatinine, uric acid was significantly increased in the hypertensive women.

Conclusion: Renal impairment is common in hypertensive pregnant women. CKD-EPI is better equation in early detection of renal impairment in pregnant women and should be adopted as the tool for assessing renal dysfunction during routine antenatal examinations to prevent end-stage renal diseases.

Keywords: Preeclampsia; Renal equations; Electrolytes; CKD-EPI

\section{Background}

Pregnancy-Induced Hypertension (PIH) is a universal problem and its global incidence is estimated between $5-14 \%$ of all pregnancies [1,2]. It is one of the major causes of pregnancy-related maternal deaths in the United States [3]. In Ghana, PIH contributes $40 \%$ of all maternal deaths [4] and one of the commonest pregnancy related complication encountered in Ghanaian hospitals.

Pregnancy-Induced Hypertension is the main cause of renal impairment especially acute kidney injury in late pregnancy, with reported incidence of between $2 \%$ and $11.3 \%$ in preeclampsia and eclampsia patients [5,6]. Chronic kidney disease (CKD) is a common complication of hypertensive patients in Ghana, with a prevalence ranging between $22 \%-46.9 \%$ among the general population $[7,8]$. Kidney disease and preeclampsia however, are caused by similar factors; both disorders have been linked to hypertension, obesity, insulin resistance and endothelial dysfunction [9-15].

A strong correlation exists between the incidence of preeclampsia and later incidence of kidney disease; moreover, preeclampsia further increases the risk for developing End-Stage Renal Disease (ESRD) [16]. Screening for renal impairment in pregnant women is not performed routinely during antenatal visits as such there is scanty information on this condition in these categories of women.

Renal equations have been widely accepted for use in the general population to offset the challenges associated with the use of the collection of 24-hour urine for the calculation of creatinine clearance.
However, the use of these equations in pregnant women still requires validation from large sample size and across different populations.

This study thus, assessed renal function in women presenting with hypertensive pregnancy using renal equations, which eliminates the cumbersome nature of requesting for 24-hour urine, with the aim of providing preliminary data that could influence the early diagnosis and management of CKD in women presenting with hypertensive pregnancy in Ghana and to encourage the adoption of these equations for routine renal assessment in women with hypertensive pregnancy.

\section{Materials and Methods}

\section{Study design and setting}

This Hospital-based case-control study was conducted at the

*Corresponding author: Linda Ahenkorah Fondjo, Department of Molecular Medicine, School of Medical Sciences, College of Health Sciences, Kwame Nkrumah University of Science and Technology, Kumasi, Ghana, Tel: +233244481606; E-mail: linda.ahenkorahfondjo@yahoo.com

Received September 28, 2017; Accepted March 13, 2018; Published March 20 2018

Citation: Fondjo LA, Owiredu WKBA, Sakyi SA, Obirikorang C, Wilfred D, et al. (2018) CKD-EPI is a Better Tool for Detecting Renal Dysfunction in Hypertensive Pregnancy: A Case-Control Study in Ghana. J Vasc Med Surg 6: 361. doi: 10.4172/2329-6925.1000361

Copyright: @ 2018 Fondjo LA, et al. This is an open-access article distributed under the terms of the Creative Commons Attribution License, which permits unrestricted use, distribution, and reproduction in any medium, provided the original author and source are credited. 
Suntreso Government Hospital in the Kumasi Metropolis between August and December 2012. The Hospital is a Government owned Primary healthcare facility located in the Ashanti Region of Ghana. It provides general health services to the public and open 24 hours. The Hospital is accredited to receive National Health Insurance as such receives a good number of patients. Kumasi is the commercial, industrial and cultural capital of the Ashanti Region and it is among the largest metropolitan cities in Ghana.

\section{Ethical consent}

The study was approved by the Committee on Human Research, Publications and Ethics (CHRPE) School of Medical Sciences Kwame Nkrumah University of Science \& Technology (KNUST) and the Institutional Review Board of Suntreso Government Hospital (CHRPE/ KNUST/RC/045/20-03-13). All willing participants enrolled in the study completed a written informed consent form. All data were coded before analysis.

\section{Selection of study participants}

A group of pregnant women receiving antenatal care at the Obstetrics and Gynecology Department of Suntreso Government Hospital were recruited for the study. In total 220 women within the age group 17-45 years were enrolled; of these 84 had Gestational Hypertension; 36 had Preeclampsia while one hundred (100) normotensive pregnant women were enrolled as controls. The diagnosis of Gestational Hypertension and Preeclampsia, was done by qualified Obstetrician/Gynecologist using the National High Blood Pressure Education Program Working Group diagnostic criteria [17]. Blood pressure of $\geq 140 / 90 \mathrm{mmHg}$ occurring after 20 weeks of gestation devoid of dipstick proteinuria was considered as Gestational Hypertension (GH) while Preeclampsia was defined as hypertension $(\geq 140 / 90)$ and proteinuria $(\geq+$ or $+>0.3 \mathrm{~g} / \mathrm{L}$ ) occurring after 20 weeks of gestation. Using closed-ended questionnaires clinical and socio-demographic data were obtained and validated through reviewing of existing records of the Hospital database.

\section{Inclusion and exclusion criteria}

Hypertensive nulliparous and multiparous Ghanaian pregnant women aged between 17-45 years and with gestational age $>20$ weeks with singleton pregnancies with or without dipstick proteinuria were enrolled as cases (PE \& GH respectively). Normotensive pregnant women without dipstick proteinuria were enrolled as controls. Respondents with previously diagnosed chronic hypertension, diabetes, heart disease, renal disease, use of antihypertensive medication before the recruitment as well as those who were unwilling to give informed consent were excluded from the study.

\section{Blood sample collection and preparation}

$4 \mathrm{~mL}$ of venous blood was taken from each respondent and allowed to clot, the serum was separated by centrifugation (Nüve NF 200, Germany) at $300 \mathrm{rpm}$ for 5 minutes within 30 minutes of collection. The obtained serum was aliquoted under sterile conditions and stored at $-80^{\circ} \mathrm{C}$ (Thermo Scientific Revco ${ }^{\mathrm{TM}} \mathrm{UxF}$ - Ultra-Low Temperature Freezers, USA) until assay.

\section{Biochemical assays}

The serum samples were analyzed for urea, creatinine and uric acid using Chemistry Analyzer, Mindray BS 380 (Shenzhen Mindray BioMedical Electronics Company Limited, China). The method employed by the automated analyser (Mindray BS 380) for the determination of the urea, creatinine and uric acid was per the reagent manufacturer's instruction- Fortress Diagnostics Limited (Fortress Diagnostics Limited, Unit 2C Antrim Technology Park, BT41 IQS United Kingdom).

The levels $\mathrm{Na}^{+}, \mathrm{K}^{+}$and $\mathrm{Cl}$ in the serum were estimated using Roche 9180 Electrolyte Analyzer (F. Hoffmann-La Roche Limited, Basel, Switzerland). The Roche 9180 Electrolyte Analyzer used employs IonSelective Electrode (ISE) measurement principles.

\section{Urine sample collection and determination of urine protein}

Participants were asked to provide about $10-15 \mathrm{~mL}$ of freshly voided morning urine in leak-proof sterile containers provided. Semi-quantitative proteinuria was determined using DIRUI A-Series Reagent dipstick Strips employing a method (DIRUI' Industrial Co. Ltd., China). Proteinuria was defined as the presence of urinary protein in concentrations $\geq$ "+", using the semi-quantitative color scale on the urine reagent dipstick [18].

\section{Measurements of anthropometric variables}

Each participant's height (to the nearest $0.1 \mathrm{~cm}$ ) was measured in standing using a wall-mounted ruler. Basal weight (weight at first trimester antenatal visit) and current weight (weight at time of recruitment) were documented for all the respondents. Their weights (to the nearest $0.1 \mathrm{~kg}$ ) were measured in kilograms in light clothing on a bathroom scale (Yongkang Yongzhou Weighing Apparatus Co. Ltd, Jinhua Zhejiang China). Basal BMI (BMI at first trimester antenatal visit) and Current BMI (BMI at time of recruitment) were estimated for all participants. BMI was calculated by dividing weight $(\mathrm{kg})$ by height squared $\left(\mathrm{m}^{2}\right)$ and documented to the nearest decimal place. Obesity: was assigned if $\mathrm{BMI} \geq 30 \mathrm{~kg} / \mathrm{m}^{2}$.

\section{Blood pressure measurement}

Participants were made to sit and rest for at least 5 minutes before the pressure was taken as recommended by the American Heart Association [19]. The blood pressure was taken by trained health personnel, using mercury sphygmomanometer with the aid of stethoscope. Duplicate measurements were taken 5 minutes apart and the mean values recorded as the blood pressure reading to the nearest $2.0 \mathrm{mmHg}$.

\section{Estimated glomerular filtration rate}

The estimated GFR (eGFR) was calculated for the pregnant women using the Chronic Kidney Disease Epidemiology Collaboration (CKDEPI) equation [20] and the Modified Diet in Renal Disease (MDRD-4) [21].

\section{Statistical analysis}

Data obtained was entered into Microsoft excel sheet and analyzed using GraphPad Prism version 5.00 for Windows (GraphPad software, San Diego California USA, www.graphpad.com). Chi-square test and Fischer's exact test for was employed for categorical data where appropriate. One-way ANOVA followed by Tukey Post-Hoc multiple comparisons were conducted between cases (PE, GH) and control. Logistic regression analysis was performed for confounding factors. The results were expressed as mean $\pm \mathrm{SD}, \mathrm{P}<0.05$ was considered as statistically significant.

\section{Results}

Socio-demographic, obstetric and clinical characteristics of study participants

The pregnant women were mostly between the ages of 30 and 39 years and married. $66.4 \%$ of the women had only basic education whilst 
Citation: Fondjo LA, Owiredu WKBA, Sakyi SA, Obirikorang C, Wilfred D, et al. (2018) CKD-EPI is a Better Tool for Detecting Renal Dysfunction in Hypertensive Pregnancy: A Case-Control Study in Ghana. J Vasc Med Surg 6: 361. doi: 10.4172/2329-6925.1000361

Page 3 of 6

$79.1 \%$ were employed in the informal sector and there was significant between-group difference in employment status $(<0.0001$ vs. $<0.0001$ vs. 0.0199). Although, most of the participants were multiparous (37.3\%), nulliparity was common among the women with preeclampsia (41.7\%) and Gestational Hypertension (35.7\%) (Table 1). More women presenting with hypertension indulged in the intake of un-prescribed herbal preparations (Table 1) however, there was no statistical difference between the groups.

\section{Biochemical characteristics}

The mean age of the control group was comparable to those presenting with hypertension (Table 2). Serum urea, creatinine, uric acid, sodium $\left(\mathrm{Na}^{+}\right)$and chloride $(\mathrm{Cl})$ concentrations were significantly increased in the GH and PE groups than in the CG (Table 3). However, there was no significant difference in their serum Potassium $\left(\mathrm{K}^{+}\right)$ levels. The two renal equations (CKD-EPI and MDRD-4) used in the estimation of the Glomerular Filtration Rate (GFR) showed that, eGFR was significantly higher in the CG than in the hypertensive cases, $(\mathrm{p}<0.001)$. Also, creatinine clearance was significantly higher in the CG compared to the GH and PE (Table 3).

\section{Renal impairments using renal equations}

Using the CKD-EPI equation the prevalence of renal impairment in

\begin{tabular}{|c|c|c|c|c|c|c|c|}
\hline Parameter & Total & Control & \#p-values & GH & $\ddagger p$-values & PE & $¥ p$-values \\
\hline & $n=220$ & $n=100$ & & $n=84$ & & $n=36$ & \\
\hline Age & & & 0.2106 & & 0.8416 & & 0.5401 \\
\hline $20-29$ & $108(49.1)$ & $53(53)$ & & $38(45.2)$ & & $17(47.2)$ & \\
\hline $30-39$ & $110(50.0)$ & $45(45)$ & & $46(54.8)$ & & $19(52.8)$ & \\
\hline $40-49$ & $2(0.9)$ & $2(2.0)$ & & $0(0.0)$ & & $0(0.0)$ & \\
\hline \multicolumn{8}{|l|}{ Marital status } \\
\hline Married & $215(97.7)$ & $97(97.0)$ & 0.797 & $82(97.6)$ & 0.3505 & $36(100.0)$ & 0.2933 \\
\hline Educational & & & 0.2789 & & 0.4934 & & 0.5351 \\
\hline No Education & $6(2.7)$ & $5(5.0)$ & & $1(1.2)$ & & $0(0.00)$ & \\
\hline Basic & $146(66.4)$ & $67(67.0)$ & & $53(63.1)$ & & $26(72.2)$ & \\
\hline Secondary & $46(20.9)$ & $20(20.0)$ & & $18(21.4)$ & & $8(22.2)$ & \\
\hline Tertiary & $22(10.0)$ & $8(8.0)$ & & $12(14.3)$ & & $2(5.6)$ & \\
\hline Employment & & & $<0.0001$ & & $<0.0001$ & & 0.0199 \\
\hline Unemployed & $14(6.4)$ & $13(13.0)$ & & $1(1.2)$ & & $0(0.00)$ & \\
\hline Informal & $174(79.1)$ & $79(79.0)$ & & 66 (78.6) & & $29(80.6)$ & \\
\hline Formal & $32(14.5)$ & $8(8.0)$ & & $17(20.2)$ & & $7(19.4)$ & \\
\hline Parity & & & 0.2707 & & 0.5468 & & 0.4763 \\
\hline Nulliparous & $74(33.6)$ & $29.0(29.0)$ & & $30(35.7)$ & & $15(41.7)$ & \\
\hline Primiparous & $62(28.2)$ & $27.0(27.0)$ & & $27(32.1)$ & & $8(22.2)$ & \\
\hline Multiparous & $82(37.3)$ & $42.0(42.0)$ & & $27(32.1)$ & & $13(36.1)$ & \\
\hline Grand Multiparous & $2.0(0.9)$ & $2.0(2.0)$ & & $0(0.0)$ & & $0(0.0)$ & \\
\hline HPI & $86(39.1)$ & $36(36.0)$ & 0.7693 & $32(38.1)$ & 0.2254 & $18(50.0)$ & 0.141 \\
\hline
\end{tabular}

Data is presented as figures with percentages in parentheses.

GH: Gestational Hypertension; PE: Preeclampsia; HPI: Herbal Preparation Intake;

\#p-Values in comparison between control and $\mathrm{GH}$;

‡p-Values in comparison between $\mathrm{GH}$ and PE;

${ }^{*} \mathrm{p}$-Values in comparison between control and PE.

Table 1: Socio-demographic characteristics of the study population.

\begin{tabular}{|c|c|c|c|c|c|c|}
\hline Parameter & Total (220) & Control (100) & GH (84) & PE (36) & $p$-value & Significant pairs \\
\hline Age (years) & $29.88 \pm 0.54$ & $29.36 \pm 0.54$ & $30.08 \pm 0.45$ & $30.86 \pm 0.60$ & 0.895 & none \\
\hline Height (cm) & $1.61 \pm 0.01$ & $1.64 \pm 0.01$ & $1.61 \pm 0.01^{* *}$ & $1.61 \pm 0.01^{*}$ & 0.0019 & Ctrl vs. GH \& PE \\
\hline Basal weight (cm) & $65.12 \pm 0.87$ & $66.02 \pm 1.27$ & $63.89 \pm 1.18$ & $65.47 \pm 2.90$ & 0.531 & none \\
\hline Current weight (kg) & $74.04 \pm 0.90$ & $72.34 \pm 1.16$ & $73.39 \pm 1.31$ & $80.28 \pm 3.10^{*}$ & 0.047 & Ctrl vs. PE \\
\hline Weight Gain (kg) & $8.92 \pm 0.39$ & $6.32 \pm 0.42$ & $9.50 \pm 0.57^{* * *}$ & $14.81 \pm 0.97^{* * *}$ & $<0.0001$ & Ctrl vs. GH \& PE \\
\hline Basal BMI & $25.23 \pm 0.35$ & $25.87 \pm 0.43$ & $24.60 \pm 0.41$ & $24.94 \pm 0.96$ & 0.852 & none \\
\hline Current BMI $\left(\mathrm{kg} / \mathrm{m}^{2}\right)$ & $28.68 \pm 0.29$ & $28.37 \pm 0.38$ & $28.25 \pm 0.44$ & $30.56 \pm 0.99$ & 0.609 & none \\
\hline BMI Gain $\left(\mathbf{k g} / \mathbf{m}^{2}\right)$ & $3.45 \pm 0.15$ & $2.50 \pm 0.17$ & $3.65 \pm 0.22^{* * *}$ & $5.62 \pm 0.36^{\star * *}$ & $<0.0001$ & Ctrl vs. GH \& PE \\
\hline SBP $(\mathrm{mmHg})$ & $141.40 \pm 2.01$ & $111.10 \pm 0.89$ & $161.40 \pm 1.06^{\star * *}$ & $178.9 \pm 1.34^{\star * \star}$ & $<0.0001$ & Ctrl vs. GH \& PE \\
\hline $\mathrm{DBP}(\mathrm{mmHg})$ & $89.05 \pm 1.64$ & $65.75 \pm 0.81$ & $101.10 \pm 0.90^{* * *}$ & $125.70 \pm 1.29^{\star \star \star}$ & $<0.0001$ & Ctrl vs. GH \& PE \\
\hline Gest. Age (weeks) & $30.38 \pm 0.29$ & $28.98 \pm 0.51$ & $30.99 \pm 0.33^{* * *}$ & $32.86 \pm 0.32^{* * *}$ & $<0.0001$ & Ctrl vs. GH \& PE \\
\hline Parity & $1.30 \pm 0.084$ & $1.59 \pm 0.51$ & $1.05 \pm 0.11^{* *}$ & $1.08 \pm 0.18$ & 0.032 & Ctrl vs. GH \\
\hline
\end{tabular}

Data is presented as mean \pm SD of the mean;

GH: Gestational Hypertension; PE: Preeclampsia; BMI: Body Mass Index;

Basal weight=weight at first antenatal visit; Current weight=weight at time of recruitment; Basal BMI=BMI at first time of antenatal visit; Current BMI=BMI at time of recruitment;

SBP: Systolic Blood Pressure; DBP: Diastolic Blood Pressure; Gest: Gestational; One-way ANOVA followed by Turkey Post Hoc multiple comparison;

${ }^{*},{ }^{* *}$ and ${ }^{* * *}$ indicates significant $p$ values at $p<0.05,<0.01$, and $<0.001$ respectively.

Table 2: Anthropometric and Gestational characteristics of respondents stratified by pregnancy outcome. 
Citation: Fondjo LA, Owiredu WKBA, Sakyi SA, Obirikorang C, Wilfred D, et al. (2018) CKD-EPI is a Better Tool for Detecting Renal Dysfunction in Hypertensive Pregnancy: A Case-Control Study in Ghana. J Vasc Med Surg 6: 361. doi: 10.4172/2329-6925.1000361

Page 4 of 6

the entire population was (4.1\%). The prevalence of renal impairment was higher in the PE group (22.2\%) compared to the GH group (1.2\%) when the CKD-EPI equation was applied. Using the MDRD-4 equation however, the prevalence of renal impairment in the study population was $0.5 \%$. None of the pregnant women in the CG had renal impairment using both equations (Table 4).

One hundred and six (106) respondents representing $48.2 \%$ were staged as having normal eGFR per the CKD-EPI formula. One hundred and five (105) respondents (47.7\%) had mild renal impairments. Nine (9) respondents $(4.1 \%)$ had moderate renal impairments (Table 5). Using the MDRD-4 formula, one hundred and six (106) representing

48.2\% had normal GFR. One hundred and thirteen (113) respondents representing $51.4 \%$ had mild renal impairment. Only (1) person was classified in stage 3 and as having moderate renal impairment. None of the respondents had severe renal impairment using both equations.

The Logistic Regression analysis indicated that neither obesity, overweight, Herbal Preparation Intake, nulliparity, primiparity nor age group 20-29 years was significant independent risk factors or predictors of preeclampsia associated renal insufficiency (Table 6).

\section{Discussion}

In this study, we assessed renal function among Ghanaian women

\begin{tabular}{|c|c|c|c|c|c|c|}
\hline Parameter & Total (220) & Control (100) & GH (84) & PE (36) & P-value & Significant pairs \\
\hline Proteinuria (g/L) & $0.08 \pm 0.02$ & $0.002 \pm 0.01$ & $0.00 \pm 0.00$ & $0.49 \pm 0.05^{* * *}$ & $<0.0001$ & Ctrl vs. GH \& PE \\
\hline Urea (mmol/L) & $4.7 \pm 0.13$ & $3.0 \pm 0.05$ & $5.5 \pm 0.13^{* * *}$ & $7.7 \pm 0.17^{\star \star \star}$ & $<0.0001$ & Ctrl vs. GH \& PE \\
\hline Uric Acid (umol/L) & $345.0 \pm 6.54$ & $264.1 \pm 5.95$ & $382.5 \pm 4.76$ & $484.8 \pm 6.54^{*}$ & 0.041 & Ctrl vs. PE \\
\hline Creatinine (umol/L) & $72.5 \pm 1.77$ & $50.4 \pm 0.88$ & $87.5 \pm 0.88^{* * *}$ & $100.8 \pm 1.77^{* * *}$ & $<0.0001$ & Ctrl vs. GH \& PE \\
\hline $\mathrm{K}^{+}(\mathrm{mmol} / \mathrm{L})$ & $3.87 \pm 0.02$ & $3.89 \pm 0.03$ & $3.83 \pm 0.03$ & $3.89 \pm 0.05$ & 0.744 & None \\
\hline $\mathrm{Na}^{+}(\mathrm{mmol} / \mathrm{L})$ & $136.70 \pm 0.11$ & $136.10 \pm 0.12$ & $137.20 \pm 0.17^{* * *}$ & $137.40 \pm 0.27^{* * *}$ & $<0.0001$ & Ctrl vs. GH \& PE \\
\hline $\mathrm{CL}^{-}(\mathrm{mmol} / \mathrm{L})$ & $102.70 \pm 0.16$ & $101.90 \pm 0.18$ & $103.20 \pm 0.27^{*}$ & $103.30 \pm 0.46^{* * *}$ & 0.0007 & Ctrl vs. GH \& PE \\
\hline CKD-EPI $\left(\mathrm{ml} / \mathrm{min} / 1.73 \mathrm{~m}^{2}\right)$ & $127.90 \pm 4.11$ & $189.50 \pm 2.92$ & $81.57 \pm 1.83^{* \star *}$ & $64.77 \pm 1.49^{* * *}$ & $<0.0001$ & Ctrl vs. GH \& PE \\
\hline $\operatorname{MDRD}^{-4}\left(\mathrm{ml} / \mathrm{min} / 1.73 \mathrm{~m}^{2}\right)$ & $112.30 \pm 2.75$ & $153.80 \pm 1.78$ & $81.59 \pm 1.29^{* \star *}$ & $68.61 \pm 1.19^{\star * *}$ & $<0.0001$ & Ctrl vs. GH \& PE \\
\hline CRCL (ml/min) & $126.80 \pm 2.61$ & $164.60 \pm 1.52$ & $97.55 \pm 2.32^{* * *}$ & $90.40 \pm 2.23^{\star * *}$ & $<0.0001$ & Ctrl vs. GH \& PE \\
\hline
\end{tabular}

Data is presented as mean \pm SD of the mean;

GH: Gestational Hypertension; PE: Preeclampsia; $\mathrm{K}^{+}$: Potassium, $\mathrm{Na}^{+}$: Sodium; $\mathrm{Cl}^{-}$: Chloride, CKD-EPI: Chronic Kidney Disease Epidemiology Collaboration; MDRD-4 Modification of Diet in Renal Disease; CRCL: Creatinine Clearance; One-way ANOVA followed by Tukey Post Hoc multiple comparison;

${ }^{*},{ }^{* *}$ and ${ }^{* * *}$ indicates significant $p$ values at $p<0.05,<0.01$, and $<0.001$ respectively.

Table 3: Biochemical characteristics of respondents stratified by pregnancy outcome.

\begin{tabular}{|c|c|c|c|c|c|c|c|c|}
\hline \multirow[t]{2}{*}{ Parameters } & \multirow{2}{*}{$\begin{array}{c}\text { Total } \\
\mathbf{n}=\mathbf{2 2 0}\end{array}$} & \multicolumn{3}{|c|}{ CT } & \multicolumn{2}{|c|}{ GH } & \multicolumn{2}{|c|}{ PE } \\
\hline & & $\%$ & $n=100$ & $\%$ & $n=84$ & $\%$ & $n=36$ & $\%$ \\
\hline \multicolumn{9}{|l|}{ CKD-EPI } \\
\hline CKD (GFR<90) & 9 & 4.1 & 0 & 0 & 1 & 1.2 & 8 & 22.2 \\
\hline NO CKD (GFR (>90) & 211 & 95.9 & 100 & 100 & 83 & 98.8 & 28 & 77.8 \\
\hline \multicolumn{9}{|l|}{ MDRD } \\
\hline CKD (GFR<90) & 1 & 0.5 & 0 & 0 & 0 & 0 & 1 & 2.8 \\
\hline NO CKD (GFR>90) & 219 & 99.5 & 100 & 100 & 84 & 100 & 35 & 97.2 \\
\hline
\end{tabular}

Data is presented as figures with percentages in parentheses;

GH: Gestational Hypertension; PE: Preeclampsia; CKD-EPI: Chronic Kidney Disease Epidemiology Collaboration; MDRD-4: Modification of Diet in Renal Disease. Table 4: Prevalence of stages of renal insufficiency among study population

\begin{tabular}{|c|c|c|c|}
\hline \multicolumn{4}{|c|}{ CKD-EPI Formula } \\
\hline \multicolumn{3}{|c|}{ STAGING } & \multirow{2}{*}{\begin{tabular}{|l|}
$\mathbf{N}(\%)$ \\
$106(48.2)$
\end{tabular}} \\
\hline $\mathbf{N}$ & Stage 1 & $>90(92-274) \mathrm{ml} / \mathrm{min} / 1.73 \mathrm{~m}^{2}$ & \\
\hline MI & Stage 2 & $60-89(60.17-88.85) \mathrm{ml} / \mathrm{min} / 1.73 \mathrm{~m}^{2}$ & $105(47.7)$ \\
\hline MOI & Stage 3 & $30-59(40.14-58.91) \mathrm{ml} / \mathrm{min} / 1.73 \mathrm{~m}^{2}$. & $9(4.1)$ \\
\hline \multirow[t]{4}{*}{ SVI } & Stage 4 & $15-29 \mathrm{ml} / \mathrm{min} / 1.73 \mathrm{~m}^{2}$ & $0(0.0)$ \\
\hline & \multicolumn{2}{|c|}{ In the hypertensive population } & \\
\hline & \multicolumn{2}{|c|}{ Total renal impairments recorded $114(95.0 \%)$} & \\
\hline & \multicolumn{2}{|c|}{ Renal disease recorded $9(7.5 \%)$} & \\
\hline \multicolumn{4}{|c|}{ MDRD-4 Formula } \\
\hline $\mathrm{N}$ & Stage 1 & $>90(91.45-206.15) \mathrm{ml} / \mathrm{min} / 1.73 \mathrm{~m}^{2}$ & $106(48.2)$ \\
\hline MI & Stage 2 & $60-89(60.46-88.05) \mathrm{ml} / \mathrm{min} / 1.73 \mathrm{~m}^{2}$ & $113(51.4)$ \\
\hline MOI & Stage 3 & $30-59(47.99) \mathrm{ml} / \mathrm{min} / 1.73 \mathrm{~m}^{2}$ & $1(0.5)$ \\
\hline \multirow[t]{4}{*}{ SVI } & Stage 4 & $15-29 \mathrm{ml} / \mathrm{min} / 1.73 \mathrm{~m}^{2}$ & $0(0.0)$ \\
\hline & \multicolumn{2}{|c|}{ In the hypertensive population } & \\
\hline & \multicolumn{2}{|c|}{ Total renal impairments recorded 114 (95.0\%) } & \\
\hline & \multicolumn{2}{|c|}{ Renal disease recorded $1(0.8 \%)$} & \\
\hline
\end{tabular}

Data is presented as figures with GFR: Glomerular Filtration rate from the smallest to the largest in parentheses

N: Normal; MI: Mild impairment; MOI: Moderate Impairment; SVI; Severe Impairment.

Table 5: Renal Impairment staging in the study population. 


\begin{tabular}{|c|c|c|}
\hline Variables & Crudes OR (95\% Cl) & p-value \\
\hline \multicolumn{3}{|l|}{ Maternal Age } \\
\hline $20-29$ & $0.527(0.1227$ to 2.267$)$ & 0.481 \\
\hline $30-39$ & ref & \\
\hline $40-49$ & - & - \\
\hline \multicolumn{3}{|l|}{ Basal BMI } \\
\hline Underweight & - & - \\
\hline Normal weight & ref & \\
\hline Overweight & $2.143(0.3810$ to 12.05$)$ & 0.3943 \\
\hline Obese & $2.857(0.1567$ to 52.09$)$ & 0.4828 \\
\hline \multicolumn{3}{|l|}{ Parity } \\
\hline Nulliparous & $0.900(0.1944$ to 4.167$)$ & 1 \\
\hline Primiparous & 0.2571 (0.024 to 2.734$)$ & 0.351 \\
\hline Multiparous & ref & \\
\hline \multicolumn{3}{|l|}{ HPI } \\
\hline Yes & 2.227 (0.5169 to 9.598$)$ & 0.4705 \\
\hline No & ref & \\
\hline
\end{tabular}

OR: Odds Ratio; Cl: Confidence Interval; HPI: Herbal Preparation Intake; ref: reference

Table 6: Logistic Regression Analysis of Predictive Factors Associated with Renal Insufficiency among PE Patients.

presenting with hypertensive pregnancy with the use of CKD-EPI and $4 \mathrm{v}-\mathrm{MDRD}$ renal equations. An overall prevalence of $4.1 \%$ and $0.5 \%$ of renal impairment was observed in the hypertensive women using CKD-EPI and MDRD equations respectively. In a case-control study, Junior et al. [6] applying the CKD-EPI equation, reported a $2 \%$ prevalence of renal impairment among Brazilian women. From this study, women with preeclampsia had a higher prevalence of renal impairment as compared to those with Gestational Hypertension using both equations (Table 4). This finding is consistent with other studies where preeclampsia has been reported to be leading cause of kidney disease in pregnancy. In a prospective observational study in India, Gopalakrishnan et al. reported preeclampsia as one of the causes of renal impairment in pregnancy [22]. In another retrospective study in Tunisia, Bouaziz et al. [23] reported preeclampsia as the leading cause of renal impairment in pregnancy. While Peng [24] in a retrospective study conducted in Beijing, suggested both GH and PE as the primary cause of renal disease. GH and PE are pregnancy specific conditions characterized by hypertension and chronic kidney disease is a known complication of Hypertension. However, in resource limited settings and in an environment where renal assessment is not routinely carried out in antenatal clinics as the case is in Ghana, the use of renal equations could become a very useful tool although its application has not been widely recognized in pregnant women.

The result of this study demonstrates that, out of the 120 hypertensive pregnant women recruited, $114(95.0 \%)$ had renal impairments using both equations (stage 2-3) (Table 5). Women with hypertensive pregnancy ( $\mathrm{GH}$ and $\mathrm{PE}$ ); the enhancement in kidney function normally experienced by pregnant women undergoing physiological pregnancies are usually repressed and these alterations in hemodynamics make the kidneys susceptible to the development of renal disease [25].

The two renal equations (CKD-EPI and MDRD-4) used to stage CKD in the respondents (Table 5), showed that, out of the 120-hypertensive pregnant women recruited, 9 (7.5\%) had moderate renal impairments when the CKD-EPI equation was used, whereas the MDRD-4 equation detected only $1(0.9 \%)$ as having moderate renal impairment (Table 5). Additionally, the CKD-EPI equation could identify more of the hypertensive women (4.1\%) as having renal disease as compared to the MDRD- 4 equation that detected only $0.5 \%$ of the study population as having renal disease.

This presupposes that CKD-EPI is a better investigative equation for early diagnosis of kidney disease in pregnant women especially in resource deprived clinical settings. Smith et al. [26] who assessed renal impairments in pregnancy complicated by renal disease or preeclampsia, suggested that in all situations, the MDRD-4 equation substantially underestimates eGFR during pregnancy and cannot be recommended for use in clinical practice. Additionally, in a Ghanaian study Eastwood et al. [27] proposed for the use of the CKD-EPI equation in assessing GFR in blacks because it was suggested as an outstanding equation when dealing with black subjects. Although, Silva Junior assessed renal function using 4 different equations, they reported that the CKD-EPI-Creatinine Cystatin C equation was a more sensitive equation to detect renal loss as it could identify $9 \%$ of their study population as having renal impairment as compared to the others that averaged $2 \%$. Moreover, not many patients in Ghana can afford cost of testing for cystatin C.

This current study further observed significantly elevated serum urea, creatinine, uric acid levels in the hypertensive group as compared to the controls (Table 3). This is likely due to the decline in clearance of these metabolites because of decline in their GFR.

CKD being a progressive clinical condition which has dare consequences for maternal and fetal mortality; can only be detected through continuous screening and close monitoring. The recommendation to screen pregnant women is an imperative approach to ensuring early detection to avert maternal and fetal complications. Therefore, using the renal equations most preferably the CKD-EPI and the staging system to define impairments in pregnant women will allow for early diagnosis of women in early stages of the disease and would aid in better diagnosis of pregnant women who are at increased risk for developing renal impairments especially CKD.

This study did not use a gold-standard assay to estimate the GFR for the comparative assessment of renal function in the pregnant women. However, this does not have any substantive effect on the findings of this study. None of the two equations used have been validated for the Ghanaian population. Also the creatinine used in the creatinine based renal function equation was not standardized to isotope dilution mass spectrophotometry (IDMS).

\section{Conclusion}

Renal impairment is prevalent $(4.1 \%)$ in hypertensive pregnant women (preeclampsia); they are at risk of developing Chronic Kidney Disease. Hence assessment of renal function using renal equation-CKDEPI would be valuable for early detection and should be incorporated in the Ghanaian antenatal protocol to help prevent End-Stage Renal Disease in this category of women.

\section{Competing Interests}

The authors declare that there is no conflict of interest regarding the publication of this paper.

\section{Authors' Contributions}

LAF and WKBAO contributed to the conception of the research idea, design, data collection, paper drafting and revision. CO, SAS and RKDE contributed to the data analysis and interpretation, paper drafting and revision. DW contributed to research design, patient recruitment, data collection, laboratory assays and paper drafting. All authors read and approved the final manuscript and agree to be responsible for all aspects of the study.

\section{Acknowledgements}

The authors are grateful to the staff and clients of the Obstetric and Gynecology Department of Suntreso Government Hospital, Kumasi Ghana for making the study possible. 
Citation: Fondjo LA, Owiredu WKBA, Sakyi SA, Obirikorang C, Wilfred D, et al. (2018) CKD-EPI is a Better Tool for Detecting Renal Dysfunction in Hypertensive Pregnancy: A Case-Control Study in Ghana. J Vasc Med Surg 6: 361. doi: 10.4172/2329-6925.1000361

Page 6 of 6

\section{References}

1. Vatten LJ, Skjaerven R (2004) Is pre-eclampsia more than one disease? BJOG: An International Journal of Obstetrics and Gynaecology 111: 298-302.

2. Karumanchi SA, Maynard SE, Stillman IE, Epstein FH, Sukhatme VP (2005) Preeclampsia: A renal perspective. Kidney International 67: 2101-2113.

3. Mustafa R, Ahmed S, Gupta A, Venuto RC (2012) A comprehensive review of hypertension in pregnancy. Journal of Pregnancy 2012: 105918.

4. Osei-Nketiah S (2001) Maternal Mortality at Effiah Nkwanta Regional Hospital, Ghana-A one Year Review (January-December), 1998. Ghana Med J 35: 124-129.

5. Peng DZ (1993) Acute renal failure in severe pregnancy induced hypertension: a report of 19 cases. Zhonghua Fu Chan Ke Za Zhi 28: 281-283.

6. Junior GSdS, Moreira SR, Nishida SK, Sass N, Kirsztajn GM (2016) Urinary abnormalities and renal function in pregnant women with chronic hypertension. Jornal Brasileiro de Nefrologia 38: 191-202.

7. Osafo C, Mate-Kole M, Affram K, Adu D (2011) Prevalence of chronic kidney disease in hypertensive patients in Ghana. Renal Failure 33: 388-392.

8. Ephraim RK, Biekpe S, Sakyi SA, Adoba P, Agbodjakey H, et al. (2015) Prevalence of chronic kidney disease among the high risk population in SouthWestern Ghana; a cross sectional study. Canadian Journal of Kidney Health and Disease 2: 40.

9. Joffe GM, Esterlitz JR, Levine RJ, Clemens JD, Ewell MG, et al. (1998) The relationship between abnormal glucose tolerance and hypertensive disorders of pregnancy in healthy nulliparous women. Calcium for Preeclampsia Prevention (CPEP) Study Group. American Journal of Obstetrics and Gynecology 179: 1032-1037.

10. Cotter AM, Molloy AM, Scott JM, Daly SF (2001) Elevated plasma homocysteine in early pregnancy: a risk factor for the development of severe preeclampsia. American Journal of Obstetrics and Gynecology 185: 781-785.

11. Jong PED, Brenner BM (2004) From secondary to primary prevention of progressive renal disease: the case for screening for albuminuria. Kidney International 66: 2109-2118.

12. Sibai BM, Gordon T, Thom E, Caritis SN, Klebanoff M, et al. (1995) Risk factors for preeclampsia in healthy nulliparous women: a prospective multicenter study. The National Institute of Child Health and Human Development Network of Maternal-Fetal Medicine Units. American Journal of Obstetrics and Gynecology 172: 642-648.

13. Owiredu WKBA, Ahenkorah L, Turpin CA, Amidu N, Laing EF (2012) Putative risk factors of pregnancy induced hypertension among Ghanaian pregnant women. The Journal of Medical and Biomedical Science 1: 62-76.

14. Turpin CA, Ahenkorah L, Owiredu WKBA, Laing EF, Amidu N (2008) The Prevalence of the Metabolic Syndrome Among Ghanaian Pregnancy-Induced
Hypertensive Patients Using the World Health Organisation and the National Cholesterol Education Program III Criteria. J Med Sci 8: 443-451.

15. Agyemang C, Oudeman E, Zijlmans W, Wendte J, Stronks K (2009) Blood pressure and body mass index in an ethnically diverse sample of adolescents in Paramaribo, Suriname. BMC Cardiovascular Disorders 9:19.

16. Vikse BE, Lorentz MI, Tobjorn L, Rolv S, Iversen BM (2008) Preeclampsia and the risk of end-stage renal disease. New England Journal of Medicine 359: 800-809.

17. Report of the National High Blood Pressure Education Program Working Group on High Blood Pressure in Pregnancy. American Journal of Obstetrics and Gynecology 183: 1-22.

18. American College of Obstetricians and Gynecologists, Task Force on Hypertension in Pregnancy (2013) Hypertension in pregnancy. Report of the American College of Obstetricians and Gynecologists' Task Force on Hypertension in Pregnancy. Obstetrics and Gynecology 122: 1122-1131.

19. Kirkendall WM, Burton AC, Epstein FH, Freis ED (1967) Recommendations for human blood pressure determination by sphygmomanometers. Circulation 36: $980-988$.

20. Levey AS, Stevens LA, Schmid CH, Zhang YL, Castro AF, et al. (2009) A new equation to estimate glomerular filtration rate. Annals of Internal Medicine 150 604-612.

21. National Kidney Foundation (2002) K/DOQI clinical practice guidelines for chronic kidney disease: evaluation, classification, and stratification. American Journal of Kidney Diseases 39: 1-266.

22. Gopalakrishnan N, Dhanapriya J, Muthukumar P, Sakthirajan R, Dineshkuma $\mathrm{T}$, et al. (2015) Acute kidney injury in pregnancy-a single center experience. Renal Failure 37: 1476-1480.

23. Bouaziz M, Chaari A, Turki O, Dammak H, Chelly H, et al. (2013) Acute renal failure and pregnancy: a seventeen-year experience of a Tunisian intensive care unit. Renal Failure 35: 1210-1215.

24. Peng $D$ (1993) [Acute renal failure in severe pregnancy induced hypertension: a report of 19 cases]. Zhonghua Fu Chan Ke Za Zhi 28: 281-283.

25. Conrad KPG, Lindheimer LW (2009) The kidney in normal pregnancy and preeclampsia. Elsevier, pp: 301-340.

26. Smith MC, Moran P, Ward MK, Davison JM (2008) Assessment of glomerular filtration rate during pregnancy using the MDRD formula. BJOG: An International Journal of Obstetrics and Gynaecology 115: 109-112.

27. Eastwood JB, Kerry SM, Plange-Rhule J, Micah FB, Antwi S (2010) Assessment of GFR by four methods in adults in Ashanti, Ghana: the need for an eGFR equation for lean African populations. Nephrology, Dialysis, Transplantation 25: 2178-2187. 